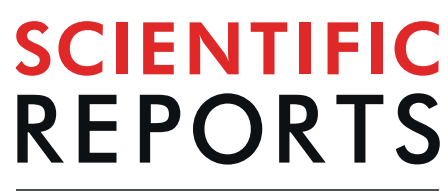

natureresearch

\title{
IL-7/IL-7R gene variants impact circulating IL-7/IL-7R homeostasis and ART-associated immune
} recovery status

\author{
Andra Ceausu ${ }^{1,11}$, Esther Rodríguez-Gallego ${ }^{1,11}$, Joaquim Peraire ${ }^{1}$, Miguel López-Dupla ${ }^{1}$, \\ Pere Domingo ${ }^{2}$, Consuelo Viladés ${ }^{1}$, Judit Vidal-Gonzalez ${ }^{3,8}$, Maria Peraire ${ }^{4,9}$, \\ Carles Perpiñán ${ }^{4,10}$, Yolanda María Pacheco ${ }^{5}$, Sergi Veloso ${ }^{1}$, Verónica Alba ${ }^{1}$, \\ Montserrat Vargas ${ }^{1}$, Alfonso J. Castellano ${ }^{1}$, Ezequiel Ruiz-Mateos ${ }^{6}$, Josep Mallolas ${ }^{7}$, \\ Francesc Vidal ${ }^{1,11 *}$ \& Anna Rull ${ }^{1,11}$
}

A relationship between polymorphisms in genes encoding interleukin 7 (IL-7) and its cellular receptor (IL-7R) and antiretroviral therapy (ART)-associated immune recovery in HIV subjects has been previously reported. However, details of this relationship remain unclear, and the association of these polymorphisms with circulating IL-7/IL-7R levels is scarce. Here, we explored whether IL-7I IL-7R axis was associated with quantitative $C D 4^{+} T$-cell recovery in HIV-infected subjects. IL-7/IL-7R polymorphisms were assessed by genotyping, and multiple inheritance models were used to estimate both, their association with low pre-ART CD4 ${ }^{+} \mathrm{T}$-cell counts and incomplete immune recovery status after 48 weeks of suppressive ART. Integrated data from genetic variants association and soluble plasma IL-7/IL-7R quantification suggest that IL-7/IL-7R genotype expression could alter the homeostatic balance between soluble and membrane-bound receptors. The haplotype analyses indicates that allele combinations impacts pre-ART circulating $\mathrm{CD4}^{+} \mathrm{T}$-cell counts, immune recovery status and the absolute increment of $\mathrm{CD}^{+}{ }^{+}$-cell counts. The knowledge about how IL-7/IL-7R axis is related to quantitative $\mathrm{CD}^{+} \mathrm{T}$-cell recovery and immune recovery status after initiating ART could be useful regarding $\mathrm{T}$-cell reservoirs investigations in HIV subjects.

Untreated HIV infection usually causes a progressive decrease in the number of circulating CD4 ${ }^{+}$T-cells over time. This may be due to underproduction and/or overdestruction of these cells ${ }^{1}$. The decrease is often profound enough to put a patient at risk of developing opportunistic infections that may ultimately lead to death. One of the objectives of antiretroviral treatment (ART) is to improve the prognosis of HIV-infected subjects due to the decrease of HIV replication below detectable levels and due to CD4 ${ }^{+}$T-cell count recovery, achieving cell counts that preclude patients from the risk of opportunistic infections. However, while this goal is achieved in a substantial proportion of patients, up to $30 \%$ of infected subjects who successfully suppress viremia below the limit of detectability do not obtain sufficient $\mathrm{CD} 4^{+} \mathrm{T}$-cell gains ${ }^{2}$. These patients are known as "poor recoverers" or "nonrecoverers"2,3, and they have worse clinical outcomes compared with "good recoverers"4-6.

\footnotetext{
${ }^{1}$ Hospital Universitari de Tarragona Joan XXIII, IISPV, Universitat Rovira i Virgili, Tarragona, Spain. ${ }^{2}$ Infectious Diseases Unit, Hospital de la Santa Creu i Sant Pau, Barcelona, Spain. ${ }^{3}$ Universitat de Barcelona, Barcelona, Spain. ${ }^{4}$ Universitat Rovira i Virgili, Tarragona, Spain. ${ }^{5}$ Laboratory of Immunology, Institute of Biomedicine of Seville, IBiS, UGC Clinical Laboratories, Virgen del Rocío University Hospital/CSIC/University of Seville, Seville, Spain. ${ }^{6}$ Clinic Unit of Infectious Diseases, Microbiology and Preventive Medicine, Institute of Biomedicine of Seville, Virgen del Rocío University Hospital/CSIC/University of Seville, Seville, Spain. ${ }^{7} \mathrm{HIV}$ Unit. Infectious Diseases Service, Hospital Clinic, Universitat de Barcelona, Barcelona, Spain. ${ }^{8}$ Present address: Servei de Medicina Interna-Hepatologia, Hospital Universitari de la Vall d'Hebron, VHIR, Barcelona, Spain. ${ }^{9}$ Present address: Hospital Universitari Son Espases, Palma de Mallorca, Spain. ${ }^{10}$ Present address: Current address: Atenció Primària ICS, Cap Sant Pere, Reus, Spain. ${ }^{11}$ These authors contributed equally: Andra Ceausu, Esther Rodríguez-Gallego, Francesc Vidal and Anna Rull. *email: fvidalmarsal.hj23.ics@gencat.cat
} 


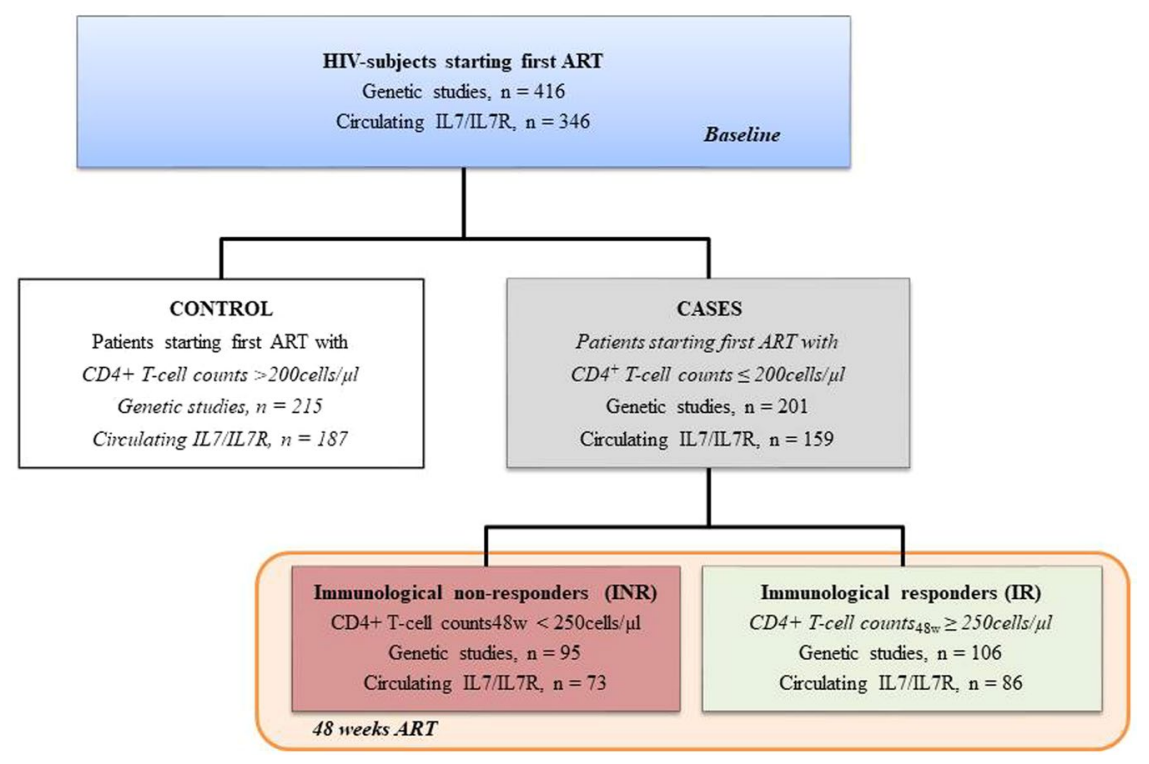

Figure 1. Flow chart illustrating subject cohort enrolment and analysis. HIV-infected subjects were included and categorized into controls and cases according to pre-ART CD4 $4^{+} \mathrm{T}$-cell counts. For an immune recovery substudy group, cases starting ART with T-cell counts below 200 cells/ $\mu \mathrm{L}$ were categorized according to their immune status after 48 weeks of follow-up.

The forces that drive the $\mathrm{CD} 4^{+} \mathrm{T}$-cell depletion and recovery dynamics are not fully known, but virus-, drugand host-related factors may all be involved. Among host factors, perturbations in molecules involved in CD4 ${ }^{+}$ T-cell homeostasis have been sought. In addition, cytokines have a key master regulatory role in T-cell homeostasis. Among them, interleukin 7 (IL-7) together with its cellular receptor (IL-7R) regulates thymic output of $\mathrm{T}_{\text {cells }}{ }^{7,8}$, promotes memory T-cell proliferation ${ }^{9}$, and mediates the survival of peripheral T-cells ${ }^{10}$. The importance of IL-7 in CD4 ${ }^{+}$T-cell turnover is highlighted by the fact that administration of recombinant human IL-7 increases $\mathrm{CD} 4^{+} \mathrm{T}$-cell counts both in patients with idiopathic CD4 ${ }^{+}$lymphocytopenia ${ }^{11}$ and in HIV-infected subjects on ART, which, despite achieving virological suppression, do not fully recover CD4 ${ }^{+}$T-cell counts ${ }^{12,13}$.

Given the essential role of IL-7 in CD4 ${ }^{+}$T-cell homeostasis, some investigations have tried to explore the association between plasma IL-7 levels and $\mathrm{CD}_{4}^{+} \mathrm{T}$-cell gain due to $\mathrm{ART}^{14-16}$ and the relationship between variations in genes encoding the IL-7/ IL-7R axis ${ }^{17-20}$. However, the results are still inconsistent, and investigations regarding the role of the IL-7/IL-7R axis are needed to understand the biologic mechanisms associated with nonrecoverers. Thus, in this study, we determined whether $I L-7$ and $I L-7 R$ single nucleotide polymorphisms (SNPs) are associated with CD4 ${ }^{+}$T-cell recovery in ART-naïve HIV-infected subjects. IL-7 and IL-7R plasma levels were also evaluated in baseline samples of HIV-infected subjects who first began ART and again after 48 and 144 weeks of follow-up under the ART regimen.

\section{Results}

Patient characteristics. The pre-ART clinical characteristics of the overall cohort of HIV-infected subjects $(\mathrm{n}=416)$ categorized according to classification criteria (Fig. 1) are presented in Table 1. Immunological nonrecoverers (INR) subjects were older, presented significantly decreased CD4 ${ }^{+} \mathrm{T}$-cell counts and increased plasma viral loads and were more likely to be diagnosed with prior AIDS-related illness. Pre-ART circulating plasma IL-7 and IL-7R values were also included for 346 samples and described below.

Genetic association study for IL-7 gene variants. Figure S1 summarizes allele and genotype frequencies for $I L-7$ gene variants located on chromosome 8 , which were in accordance with data listed on the NCBI SNP database. The genotype frequencies for $r s 6987789$ and $r s 7007634$ were consistent with HWE (Figure S1-B). After verifying that there was no association between these $I L-7$ gene variants and low pre-ART CD4 ${ }^{+}$T-cell counts $\left(\mathrm{CD} 4^{+} \mathrm{T}\right.$-cell $\leq 200$ cells $\left./ \mu \mathrm{L}\right)$ (Figure S1-C, cases versus controls), we investigated whether there was any association with poor ART-associated immune response (Figure S1-C, INR versus immunological recoverers (IRs)). No association was found between these $I L-7$ gene variants and incomplete immune recovery status. Finally, in the multiple-SNP analysis, linkage disequilibrium (LD) was found between $r s 6987789$ and $r s 7007634$ $\left(D^{\prime}=0.2713, r=0.0982, P=0.055\right)$. Any of the possible haplotypes were related to ART-associated immune recovery (Table $\mathrm{S} 1$ ).

Association between rs10491434 (IL-7R) and low pre-ART CD4+ T-cell counts. Figure S2 summarizes allele frequencies for $I L-7 R$ gene variants on chromosome 5 , which were in accordance with data listed on the NCBI SNP database. The genotype frequencies for the $I L-7 R$ gene variants explored in this study were consistent with HWE, except for $r s 969128$ (Table S2). Then, we searched for an association between the $I L$ $7 R$ gene variants and low pre-ART CD4 ${ }^{+}$T-cell counts. Considering Akaike's Information Criteria (AIC) and 


\begin{tabular}{|c|c|c|c|c|}
\hline & \multicolumn{2}{|c|}{$\mathrm{CD}^{+}{ }^{\mathrm{T}}$-cells $\leq \mathbf{2 0 0}$ cells $/ \mu \mathrm{L}$} & \multirow{2}{*}{\begin{tabular}{|l|} 
CD4 $^{+}$T-cells $>200$ cells $/ \mu \mathrm{L}$ \\
Control $(n=215)$
\end{tabular}} & \multirow[b]{2}{*}{ P-value } \\
\hline & INR $(n=95)$ & $\operatorname{IR}(n=106)$ & & \\
\hline \multicolumn{5}{|l|}{ Pre-ART clinical characteristics } \\
\hline Age at cART initiation (years) & $42[37-51]$ & $37[33-42]$ & 37 [31-45] & $<0.001$ \\
\hline Male & $58(79)$ & $70(82)$ & $155(83)$ & 0.807 \\
\hline Risk factor & & & & 0.060 \\
\hline Heterosexual & $29(40)$ & $31(36)$ & $62(33)$ & \\
\hline Homo/Bisexual & $26(36)$ & $47(55)$ & $101(54)$ & \\
\hline Intravenous drug abuse & $15(20)$ & $6(7)$ & $19(10)$ & \\
\hline Other/Unknown & $3(4)$ & $2(2)$ & $5(3)$ & \\
\hline $\mathrm{CD}^{+}{ }^{+} \mathrm{T}$-cell count (cells/ $\left.\mu \mathrm{L}\right)$ & $51[17-111]$ & $129[51-181]$ & 333 [272-441] & $<0.001$ \\
\hline Plasma HIV RNA load (log copies/mL) & $5.30[4.82-5.62]$ & $5.06[4.71-5.61]$ & $4.87[4.33-5.25]$ & $<0.001$ \\
\hline AIDS indicator condition & $30(41)$ & $21(24)$ & $13(7)$ & $<0.001$ \\
\hline HCV co-infection (Positive) & $13(20)$ & $10(12)$ & $27(15)$ & 0.410 \\
\hline \multicolumn{5}{|l|}{ ART clinical characteristics } \\
\hline 48 weeks $\mathrm{CD} 4^{+} \mathrm{T}$-cell count (cells/ $\left.\mu \mathrm{L}\right)$ & $167[110-216]$ & 350 [294-422] & 532 [408-699] & $<0.001$ \\
\hline$\Delta \mathrm{CD} 4^{+} \mathrm{T}$-cell count & 95 [47-142] & 239 [187-316] & 170 [64-289] & $<0.001$ \\
\hline Pre-ART plasma IL-7/IL-7R $(n=345)$ & $(n=73)$ & $(\mathbf{n}=\mathbf{8 6})$ & $(n=187)$ & \\
\hline Log IL-7 (pg/mL) & $2.65[2.53-2.82]$ & $2.67[2.54-2.81]$ & $2.72[2.60-2.99]$ & 0.037 \\
\hline Log IL-7R (ng/mL) & $2.34[2.02-2.49]$ & $2.33[2.08-2.54]$ & $2.34[2.16-2.63]$ & 0.264 \\
\hline
\end{tabular}

Table 1. Study cohort $(n=416)$ characteristics of the according classification criteria. Data are presented as $n$ (\%) or median (interquartile range). Categorical data were compared by means of a $\chi^{2}$ test, whereas continuous data were compared using non-parametric Kruskall-Wallis test. P value $<0.05$ was considered significant and is highlighted in bold. All $\mathrm{P}$ values $>0.05$ but $<0.15$ were considered relevant for results interpretation and are italicized. AIDS was diagnosed according the CDC1993 criteria. INR, incomplete immune recoverers; IR, immune recoverers.

Bayesian Information Criteria (BIC) scores and adjusting for age and baseline pre-ART CD4 ${ }^{+}$T-cell counts, an association was only detected with the overdominant model for $r$ s10491434 (Table S3). In the multiple-SNP analysis (Figure S3 and Table S4), LD was found between several genetic variants (Figure S3), and the haplotype TAGAGCTCTAAT, which is present in $16 \%$ of the study cohort, was related to low pre-ART CD4 ${ }^{+}$T-cell counts (Table S4-B).

Two haplotypes from the $I L-7 R$ gene variants associated with immune restoration. Next, we explored the association between $I L-7 R$ gene variants and immune recovery status. Considering AIC and BIC scores and adjusting for age and baseline pre-ART CD4 ${ }^{+} \mathrm{T}$-cell counts, the best associations were with the recessive models for $r s 1494558(\mathrm{OR}=0.16,95 \% \mathrm{CI}=0.05-0.57, \mathrm{P}=0.0017)$, $r s 969129(\mathrm{OR}=0.29,95 \% \mathrm{CI}=0.09$ $0.91, \mathrm{P}=0.025)$ and $r s 1494555(\mathrm{OR}=0.20,95 \% \mathrm{CI}=0.06-0.67, \mathrm{P}=0.0044)$ (Table 2). LD was found between several gene variants (Fig. 2), and the haplotypes TAGAGCTCCAGC $(\mathrm{OR}=1.86,95 \% \mathrm{CI}=1.02-3.37, \mathrm{P}=0.04)$ and TGGGGCTTCTAT $(\mathrm{OR}=2.46,95 \% \mathrm{CI}=1.05-5.72, \mathrm{P}=0.039)$ were associated with the immune response according to $\mathrm{CD} 4^{+}$T-cell counts after 48 weeks of ART (Table S5, Fig. 2).

Association between circulating IL-7 values and pre-ART CD4 ${ }^{+}$T-cell counts. Pre-ART circulating IL-7/IL-7R values were evaluated in 346 plasma samples, including 187 controls and 159 cases (73 INRs and 86 IRs). The cases revealed lower circulating IL-7 values than the controls $[2.66[2.53-2.81] \log \mathrm{pg} / \mathrm{mL}$ and 2.72 [2.60-2.98] $\log \mathrm{pg} / \mathrm{mL}$, respectively, $\mathrm{P}=0.03]$. In fact, correlation analysis indicated a positive association between circulating IL-7 values and low pre-ART CD4 ${ }^{+}$T-cell counts $(\rho=0.106, \mathrm{P}=0.045)$. No differences were observed in IL-7R values. However, IL-7R values were significantly associated with circulating IL-7 values $(\rho=0.692, \mathrm{P}<0.001)$, a correlation that was consistent in the cases $(\rho=0.683, \mathrm{P}<0.001)$ and the controls $(\rho=0.693, \mathrm{P}<0.001)$.

Then, we explored whether changes in the IL-7/IL-7R axis could predict ART-associated immune recovery status. No differences in pre-ART circulating IL-7/IL-7R values were observed between INRs and IRs (Table 1). When correlation analyses were performed among the different groups, we observed a positive association between IL-7R values and the $\Delta \mathrm{CD}^{+} \mathrm{T}$-cell counts $(\rho=0.246, \mathrm{P}=0.023)$ in IRs.

IL-7R gene variants impact circulating IL-7R in IRs. The plasma IL-7/IL-7R axis was not influenced by any of the $I L-7$ and $I L-7 R$ SNPs analyzed. However, when the impact of $I L-7 / I L-7 R$ gene variants was explored in INRs and IRs individually, IRs revealed a significant association between circulating plasma IL-7R concentrations and $r s 987106, r s 3194051$, and $r s 10491434$ (Fig. 3). A strong LD among these IL-7R SNPs (Fig. 2, D'>0.95) was found, meaning that these alleles of each gene are inherited together more often than would be expected by chance. 


\begin{tabular}{|c|c|c|c|c|c|c|c|c|}
\hline Polymorphism & IHT model & Genotype & INR & IR & OR $(95 \% \mathrm{CI})$ & P-value & AIC & BIC \\
\hline \multirow{2}{*}{ rs7701176 T > A } & \multirow{2}{*}{-} & $\mathrm{T} / \mathrm{T}$ & $91(97.8 \%)$ & $104(100 \%)$ & 1.00 & \multirow{2}{*}{0.083} & \multirow{2}{*}{230.1} & \multirow{2}{*}{243.2} \\
\hline & & T/A & $2(2.1 \%)$ & $0(0 \%)$ & $0.00(0.00-\mathrm{NA})$ & & & \\
\hline \multirow{2}{*}{$\mathrm{rs} 1494559 \mathrm{~A}>\mathrm{G}$} & \multirow{2}{*}{ Dominant } & $\mathrm{A} / \mathrm{A}$ & $66(77.7 \%)$ & $68(68.7 \%)$ & 1.00 & \multirow{2}{*}{0.420} & \multirow{2}{*}{220.7} & \multirow{2}{*}{233.6} \\
\hline & & G/A-G/G & $19(22.4 \%)$ & $31(31.3 \%)$ & $1.35(0.65-2.83)$ & & & \\
\hline \multirow{2}{*}{ rs $1494558 \mathrm{G}>\mathrm{A}$} & \multirow{2}{*}{ Recessive } & G/G-G/A & $68(81 \%)$ & $94(95.9 \%)$ & 1.00 & \multirow{2}{*}{0.002} & \multirow{2}{*}{205.8} & \multirow{2}{*}{218.6} \\
\hline & & $\mathrm{A} / \mathrm{A}$ & $16(19.1 \%)$ & $4(4.1 \%)$ & $0.16(0.05-0.57)$ & & & \\
\hline \multirow[b]{2}{*}{ rs969128 A > G } & \multirow[b]{2}{*}{ Dominant } & $\mathrm{A} / \mathrm{A}$ & $64(79 \%)$ & $70(68 \%)$ & 1.00 & \multirow{2}{*}{0.240} & \multirow{2}{*}{211.3} & \multirow{2}{*}{224.2} \\
\hline & & G/A-G/G & $17(21 \%)$ & $33(32 \%)$ & $1.57(0.74-3.36)$ & & & \\
\hline \multirow{2}{*}{ rs969129 G > T } & \multirow{2}{*}{ Recessive } & G/G-T/G & $71(82.6 \%)$ & $93(94.9 \%)$ & 1.00 & \multirow{2}{*}{0.025} & \multirow{2}{*}{214} & \multirow{2}{*}{226.9} \\
\hline & & $\mathrm{T} / \mathrm{T}$ & $15(17.4 \%)$ & $5(5.1 \%)$ & $0.29(0.09-0.91)$ & & & \\
\hline \multirow{2}{*}{ rs6893892 C > T } & \multirow{2}{*}{-} & $\mathrm{C} / \mathrm{C}$ & 92 (98.9\%) & $100(99 \%)$ & 1.00 & \multirow{2}{*}{0.970} & \multirow{2}{*}{231.5} & \multirow{2}{*}{244.6} \\
\hline & & $\mathrm{T} / \mathrm{C}$ & $1(1.1 \%)$ & $1(1 \%)$ & $1.07(0.06-18.78)$ & & & \\
\hline \multirow{2}{*}{$\mathrm{r} 1494555 \mathrm{~T}>\mathrm{C}$} & \multirow{2}{*}{ Recessive } & T/T-C/T & $70(80.5 \%)$ & $90(94.7 \%)$ & 1.00 & & & \\
\hline & & $\mathrm{C} / \mathrm{C}$ & $17(19.5 \%)$ & $5(5.3 \%)$ & $0.20(0.06-0.67)$ & 0.004 & 203 & 215.9 \\
\hline $\mathrm{rc} 2228141 \mathrm{C}>\mathrm{T}$ & Dominant & $\mathrm{C} / \mathrm{C}$ & $72(80 \%)$ & $68(68 \%)$ & 1.00 & 0250 & 224 & 237 \\
\hline rS $22 \angle 8141 \mathrm{C}>1$ & Dominant & T/C-T/T & $18(20 \%)$ & $32(32 \%)$ & $1.54(0.73-3.22)$ & 0.250 & 224 & 237 \\
\hline rc6897032 $C>\mathrm{T}$ & Peсескіу & C/C-T/C & $81(94.2 \%)$ & 99 (99\%) & 1.00 & 0 & 2215 & 2344 \\
\hline rss689/932C > 1 & Recessive & $\mathrm{T} / \mathrm{T}$ & $5(5.8 \%)$ & $1(1 \%)$ & $0.17(0.02-1.63)$ & 0.082 & 221.5 & 234.4 \\
\hline $\mathrm{rc} 987106 \mathrm{~T}>\mathrm{A}$ & Dominant & $\mathrm{T} / \mathrm{T}$ & $31(34.1 \%)$ & $24(23.8 \%)$ & 1.00 & & & \\
\hline rs987106 T > A & Dominant & T/A-A/A & $60(65.9 \%)$ & $77(76.2 \%)$ & $1.69(0.84-3.43)$ & 0.140 & 225.9 & 238.9 \\
\hline 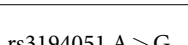 & Dominnnt & $\mathrm{A} / \mathrm{A}$ & $47(53.4 \%)$ & $46(46.5 \%)$ & 1.00 & 020 & 217 & 2209 \\
\hline rs3194051 A > G & Dominant & G/A-G/G & $41(46.6 \%)$ & $53(53.5 \%)$ & $1.54(0.79-2.99)$ & 0.200 & 217 & 229.9 \\
\hline$r s 10491434 \mathrm{~T}>C$ & Dominant & $\mathrm{T} / \mathrm{T}$ & $46(53.5 \%)$ & 45 (45.9\%) & 1.00 & & & 2268 \\
\hline rs10491434 I >C & Dominant & T/C-C/C & $40(46.5 \%)$ & $53(54.1 \%)$ & $1.63(0.83-3.18)$ & 0.150 & 213.9 & 226.8 \\
\hline
\end{tabular}

Table 2. $I L-7 R$ SNP association with poor immune recovery after 48 weeks of cART. The logistic regression model was adjusted by age and pre-ART CD4 ${ }^{+}$T-cell counts. Data analysis is summarized with n (\%), odds ratio (OR) and 95\% confidence interval (CI) for each SNP. P value of the likehood ratio test less than $<0.05$ was considered significant and is highlighted in bold. The AIC and BIC values were used to choose the inheritance model (IHT) that best fits the data. AIC; Akaike's Information Criteria; BIC; Bayesian Information Criteria.

rs3194051 (IL-7R) influences the absolute increment of CD4+T-cell counts. Because the $\Delta \mathrm{CD} 4^{+}$ T-cell count was significantly lower in INRs (Table 1), we also decided to verify any possible association between the $\Delta \mathrm{CD} 4^{+}$T-cell count and the IL-7/IL-7R axis. Therefore, all subjects $(\mathrm{n}=416)$ were reclassified in two groups according $\Delta \mathrm{CD} 4^{+}$T-cell count after 48 weeks of ART (Fig. 4A), and those with a $\Delta \mathrm{CD} 4^{+}$T-cell count $<100$ cells/ $\mu \mathrm{L}(\mathrm{n}=136)$ were considered poor immune recoverers and were clearly differentiated from immune recoverers (Fig. 4B).

First, no association was found between $I L-7$ gene variants and $\triangle \mathrm{CD} 4^{+} \mathrm{T}$-cell counts. Any of the possible haplotypes were related to these absolute increments (global P-value $=0.44$, Table S6). However, regarding the $I L-7 R$ gene variants, the best associations were with the recessive models for $r s 3194051(\mathrm{OR}=2.51,95 \% \mathrm{CI}=0.97$ 6.48, $\mathrm{P}=0.04$ ) (Table S7). LD was found between several gene variants (data not shown), and the haplotype TAGAGCTCCAGC, which was previously associated with immune response (Fig. 2), was also associated with a $\Delta \mathrm{CD} 4^{+} \mathrm{T}$-cell count $>100$ cells/ $\mu \mathrm{L}$ (Fig. 4C).

Second, according to this classification criterion, subjects with $\Delta \mathrm{CD} 4^{+} \mathrm{T}$-cell counts $<100$ cells/ $\mu \mathrm{L}$ after 48 weeks of ART regimen revealed decreased circulating IL-7/IL-7R values (Fig. 4D).

Longitudinal evaluation of IL-7/IL-7R plasma concentrations. First, we examined plasma IL-7/ IL-7R concentrations at different time points during the 48 weeks of the ART regimen. We also included data from 197 HIV-infected subjects with available follow-up samples after 144 weeks on ART. Circulating IL-7 concentrations remained lower in the cases than the controls during the 144 weeks of ART. By contrast, IL-7R values became similar until 48 weeks of ART, when IL-7R values were again lower in the cases than the controls (Fig. 5A). No differences were observed among INRs and IRs (data not shown).

Finally, we evaluated the association between IL-7/IL-7R parameters and $\Delta \mathrm{CD} 4^{+}$T-cell. Circulating IL-7 concentrations remained lower in subjects with a $\Delta \mathrm{CD} 4^{+} \mathrm{T}$-cell count $<100$ cells $/ \mu \mathrm{L}$, with significant differences at 48 weeks of ART. In addition, circulating IL-7R values also remained lower in subjects with a $\Delta \mathrm{CD} 4{ }^{+} \mathrm{T}$-cell counts $<100$ cells $/ \mu \mathrm{L}$, and these differences were also significant after 48 and 144 weeks of ART (Fig. 5B).

\section{Discussion}

This is the first study to assess both the association of IL-7 genetic background and circulating IL-7 behavior overtime with quantitative immune recovery in treated HIV-infected patients. Some previous evidence suggests that genetic variation within the IL-7/IL-7R axis may modulate the magnitude of CD4 ${ }^{+}$T-cell gains ${ }^{17-20}$, although the underlying mechanisms remain unclear. On the other hand, whereas an increase in IL-7 production has 
A

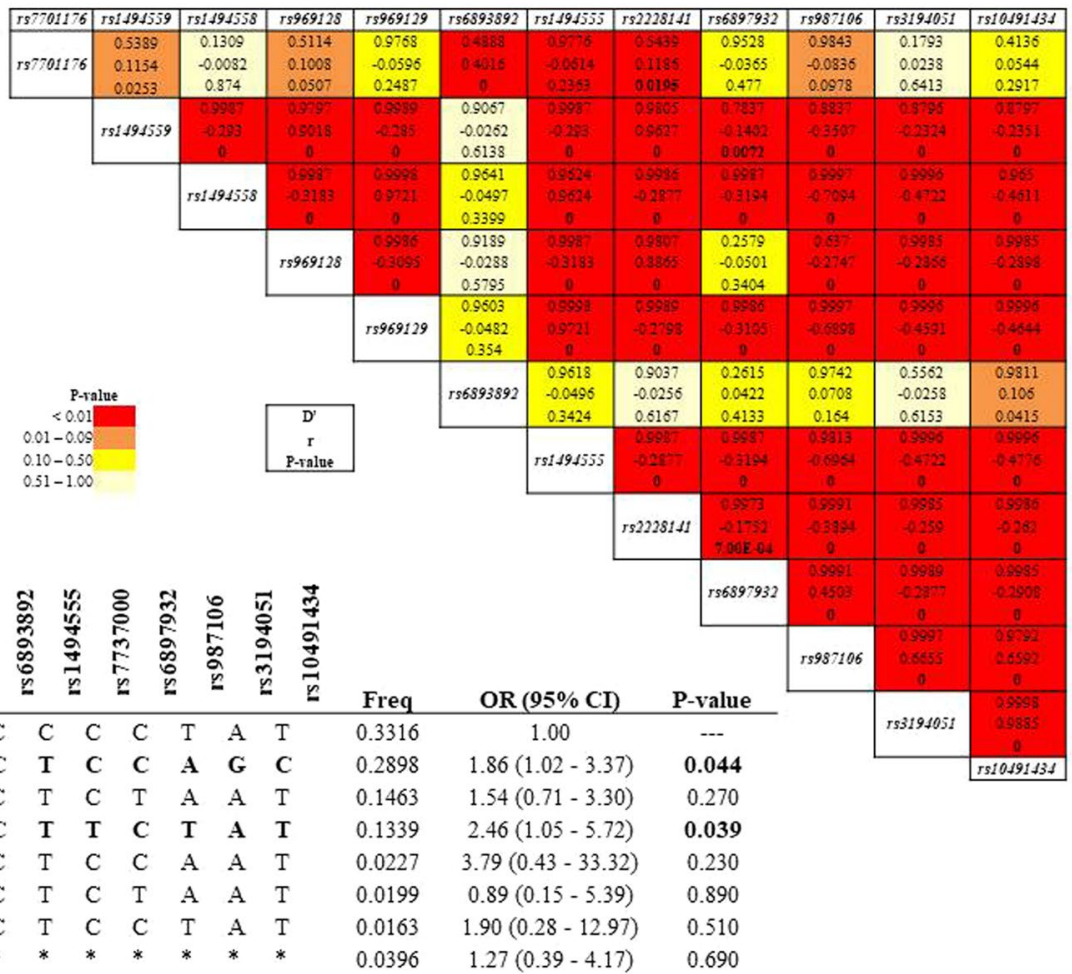

B

\begin{tabular}{|c|c|c|c|c|c|c|c|c|c|c|c|c|c|c|c|c|c|}
\hline & 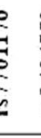 & 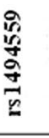 & 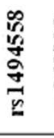 & 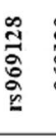 & 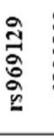 & 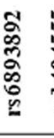 & 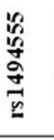 & $\begin{array}{l}\stackrel{8}{\circ} \\
\stackrel{8}{2} \\
2 \\
2\end{array}$ & 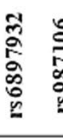 & $\begin{array}{l}0 \\
\vdots \\
\vdots \\
\vdots \\
2 \\
2\end{array}$ & 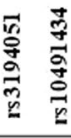 & Freq & OR $(95 \% \mathrm{CI})$ & rs6597932 & $\begin{array}{c}04503 \\
0 \\
r s 987106\end{array}$ & $\begin{array}{c}-0287 \\
0 \\
09992 \\
0.5655 \\
0 \\
\end{array}$ & $\begin{array}{c}-0.2005 \\
0 \\
0.5597 \\
0.6592 \\
0 \\
0.5995\end{array}$ \\
\hline $\mathrm{T}$ & $\mathrm{A}$ & $\mathrm{A}$ & $\mathrm{A}$ & $\mathrm{T}$ & $\mathrm{C}$ & $\mathrm{C}$ & $\mathrm{C}$ & $\mathrm{C}$ & $\mathrm{T}$ & $\mathrm{A}$ & $\mathrm{T}$ & 0.3316 & 1.00 & -- & & rssig405i & 09885 \\
\hline $\mathbf{T}$ & A & G & $\mathbf{A}$ & G & C & $\mathrm{T}$ & C & $\mathrm{C}$ & $A$ & G & C & 0.2898 & $1.86(1.02-3.37)$ & 0.044 & & & rsitod91434 \\
\hline $\mathrm{T}$ & A & G & A & G & $\mathrm{C}$ & $\mathrm{T}$ & $\mathrm{C}$ & $\mathrm{T}$ & A & A & $\mathrm{T}$ & 0.1463 & $1.54(0.71-3.30)$ & 0.270 & & & \\
\hline $\mathbf{T}$ & G & G & G & G & $\mathrm{C}$ & $\mathbf{T}$ & $\mathbf{T}$ & $\mathrm{C}$ & $\mathbf{T}$ & $A$ & $\mathbf{T}$ & 0.1339 & $2.46(1.05-5.72)$ & 0.039 & & & \\
\hline $\mathrm{T}$ & A & G & $\mathrm{A}$ & G & $\mathrm{C}$ & $\mathrm{T}$ & $\mathrm{C}$ & $\mathrm{C}$ & A & $A$ & $\mathrm{~T}$ & 0.0227 & $3.79(0.43-33.32)$ & 0.230 & & & \\
\hline $\mathrm{T}$ & A & G & G & G & $\mathrm{C}$ & $\mathrm{T}$ & $\mathrm{C}$ & $\mathrm{T}$ & A & $\mathrm{A}$ & $\mathrm{T}$ & 0.0199 & $0.89(0.15-5.39)$ & 0.890 & & & \\
\hline $\mathrm{T}$ & $\mathrm{A}$ & A & A & G & $\mathrm{C}$ & $\mathrm{T}$ & $\mathrm{C}$ & $\mathrm{C}$ & $\mathrm{T}$ & $\mathrm{A}$ & $\mathrm{T}$ & 0.0163 & $1.90(0.28-12.97)$ & 0.510 & & & \\
\hline$*$ & * & $*$ & $*$ & $*$ & $*$ & * & $*$ & * & * & * & * & 0.0396 & $1.27(0.39-4.17)$ & 0.690 & & & \\
\hline
\end{tabular}

Global haplotype association P-value: 0.34

Figure 2. Haplotype analysis for the $I L-7 R$ gene variants explored in this study. (A) Linkage disequilibrium (LD) analysis in INR subjects compared to IR subjects for $I L-7 R$ gene variants. (B) Haplotype association with immune recovery status according to CD4 ${ }^{+}$T-cell counts after 48 weeks of ART $(n=197)$.

been proposed as part of the homeostatic response to T-cell depletion ${ }^{16}$ and thus relates lower pre-ART IL-7 levels to faster $\mathrm{CD} 4^{+} \mathrm{T}$ cell recovery ${ }^{18}$, other studies suggest an association between lower concentrations of the IL-7/IL-7R axis with an insufficient ability to restore the number of CD4 $4^{+} \mathrm{T}$ cells in response to ART ${ }^{14,21}$. In this longitudinal study, we demonstrated that IL-7R gene variants were associated with low pre-ART CD4 ${ }^{+} \mathrm{T}$ cell counts and immune restoration according to immune recovery status after 48 weeks on ART. Furthermore, our data revealed that IL-7R gene variants impact pre-ART IL-7R plasma concentrations and the absolute increment of $\mathrm{CD}^{+}$T cell counts during ART. Circulating pre-ART IL-7 levels were low in HIV-infected subjects with low pre-ART CD4 ${ }^{+}$T cell counts and significantly correlated with pre-ART IL-7R plasma concentrations. In addition, we observed that IL-7/IL-7R values remained lower in subjects with an increment of CD4 ${ }^{+}$T-cell counts of less than $100 \mathrm{CD}^{+} \mathrm{T}$-cells/ $\mu \mathrm{L}$ during the follow-up until 144 weeks of ART than in those with $\Delta \mathrm{CD} 4^{+} \mathrm{T}$-cell counts greater than 100 cells/ $\mu \mathrm{L}$. To our knowledge, this study is the first description of the relationship between IL-7/ IL-7R polymorphisms and pre-ART IL-7/IL-7R plasma concentration in relation to low pre-ART CD4 ${ }^{+}$T-cell counts and immune restoration due to ART.

First, no association was found between IL-7 gene variants and low pre-ART CD4 ${ }^{+}$T-cell counts, immune restoration or absolute increment of $\mathrm{CD} 4^{+}$T-cell counts due to ART. Similar to our data, in a previous case-controlled association study exploring the influence of the IL-7/IL-7R pathway on HIV-1 pathogenesis and AIDS progression, no association with IL-7 gene variants could be found ${ }^{22}$. Notice that IL-7 is a nonredundant cytokine that is crucial for B- and T-cell homeostasis that mediates a plenitude of functions in health and disease $^{23}$. Therefore, its importance for the survival and development and proliferation of B- and T-cells could explain the low levels of IL-7 gene diversity ${ }^{24}$, which was also previously observed in multiple sclerosis (MS) ${ }^{25}$.

Second, the $r s 10491434$ variant of the IL-7R gene and the H3 haplotype tagged by $r$ s 10491434 -T (17\% controls versus $14 \%$ cases) were associated with pre-ART CD4 ${ }^{+}$T-cell counts. $r s 10491434$ has been previously implicated in AIDS progression ${ }^{22}$, and the $r s 10491434 \mathrm{~T} / \mathrm{T}$ genotype is associated with HIV subjects that achieve CD4 ${ }^{+} \mathrm{T}_{\text {-cell }}$ counts higher than $500 \mathrm{CD} 4^{+} \mathrm{T}$-cells/ $\mu \mathrm{L}$ after 48 months on $\mathrm{ART}^{26}$. Consistent with this, in our study, patients with $r s 10491434 \mathrm{~T} / \mathrm{T}-\mathrm{C} / \mathrm{C}$ genotypes were more closely associated with high pre-ART CD4 ${ }^{+} \mathrm{T}$-cell counts $\left(\mathrm{CD} 4^{+}\right.$ T-cells $>200$ cells $/ \mu \mathrm{L}$ ) before initiating ART therapy than were patients with $r s 10491434 \mathrm{~T} / \mathrm{C}$ genotypes. Notably, rs 10491434 is located at the $3^{\prime}$ UTR, showing allele-specific methylation at nearby CpG sites ${ }^{27}$; more specifically, the $r s 10491434 \mathrm{~T}$ allele associated with lower methylation at nearby CpG sites increases IL-7R expression ${ }^{26}$. In fact, in our study, we found a significant association between $r s 10491434$ and circulating pre-ART IL-7R values in IRs. Concretely, IR subjects with the rs10491434 TT genotype (major allele homozygous) showed higher plasma IL-7R values than did patients with the $r$ s10491434 TC/CC genotype. Additionally, circulating IL-7R values revealed a positive correlation with the absolute increment of $\mathrm{CD} 4^{+} \mathrm{T}$-cell counts in this subgroup of subjects, corroborating the association between this gene variant and $\mathrm{CD} 4^{+} \mathrm{T}$-cell recovery. Additionally, this 

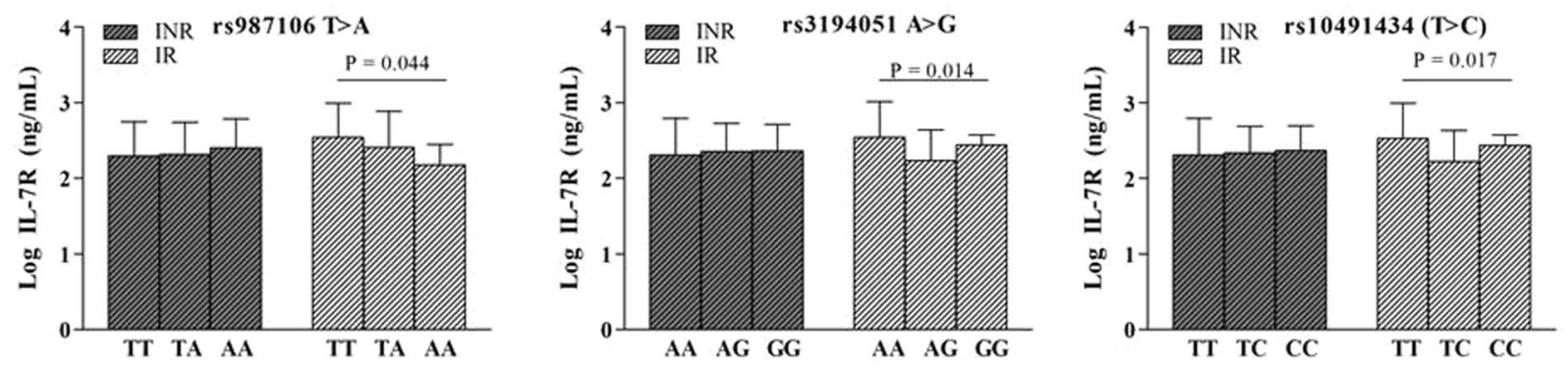

Figure 3. Influence of $I L-7 R$ genes in circulating concentrations of IL-7R. Rs987106, $r s 3194051$ and $r s 10491434$ variants influence circulating plasma concentrations in IR subjects. Data are represented as the mean \pm SD $(65$ INRs and 75 IRs). The relationship between $I L-7 R$ genes and plasma IL-7 concentrations were analyzed using one-way ANOVA test (SPSS software).

A

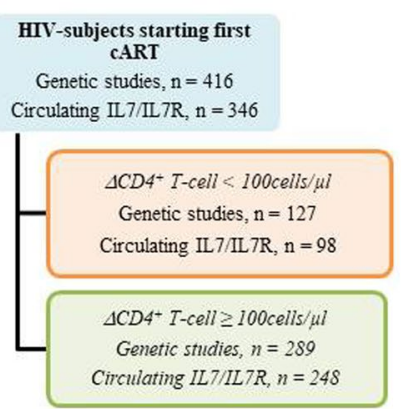

B

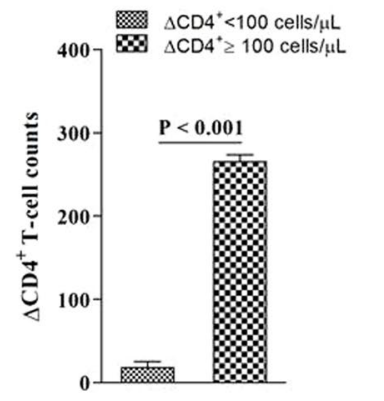

C
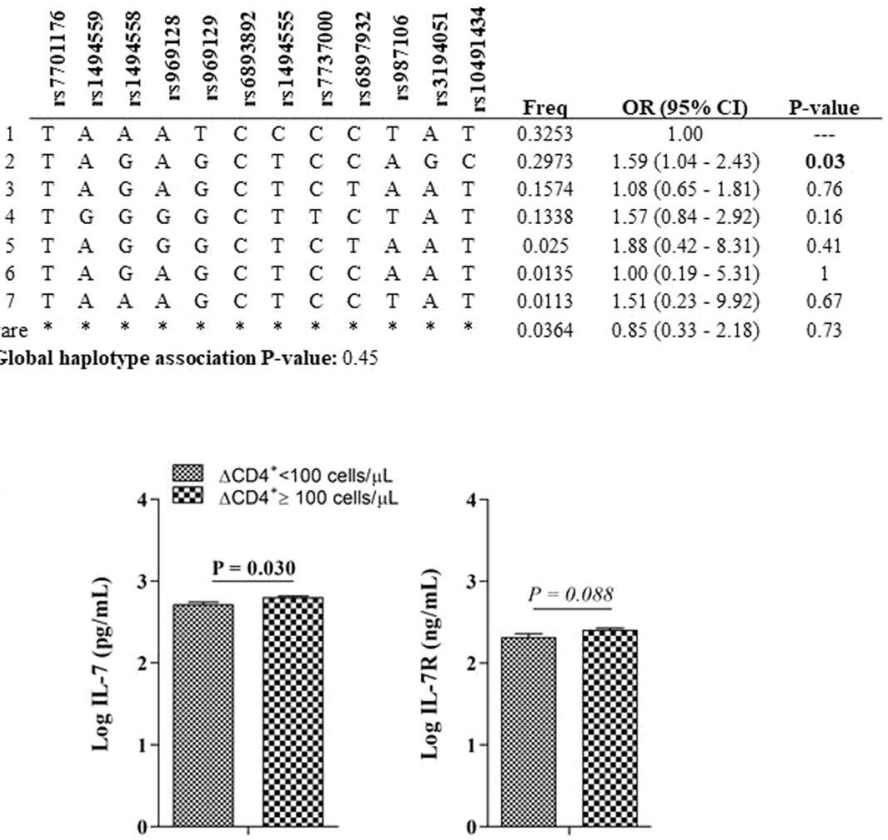

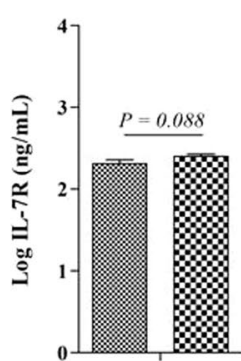

Figure 4. Impact of IL-7/IL-7R axis on the absolute increment of CD4+ T-cell counts. (A) Flow chart illustrating classification criteria according the absolute increment of CD4+ T-cell counts $(\Delta \mathrm{CD} 4+\mathrm{T}$-cell) after 48 weeks of ART. (B) $\Delta \mathrm{CD} 4^{+} \mathrm{T}$-cell count mean $\pm \mathrm{SD}$ values in cases 2 and controls 2 according to this classification criterion $(\mathrm{n}=416)$. (C) Haplotype association with the $\Delta$ CD4+T-cell count $(\mathrm{n}=452)$. (D) PreART circulating concentrations of IL-7 $(\mathrm{n}=356)$ and IL-7R $(\mathrm{n}=349)$ according $\Delta$ CD4+ T-cell count criteria. Data are represented as the mean $\pm \mathrm{SD}$. Comparisons between groups were performed with nonparametric Mann-Whitney (MW) (SPSS software).

$3^{\prime}$ gene region polymorphism is in LD with several SNPs that could impact the function of the IL-7 receptor, among which are the intronic SNP $r s 987106$ and the exonic SNP $r s 3194051$. In this sense, not only the $r s 10491434$ genotype impacts circulating IL-7R concentrations in IRs but also the $r s 987106$ and $r s 319405$ genotypes seem to influence pre-ART circulating IL-7R concentrations. In the case of the $r s 987106$ gene variant, IRs carrying the T-allele revealed higher IL-7R values compared with those of the patients carrying the A-allele, in accordance with previous data ${ }^{19}$. $r s 987106-\mathrm{T}$ was previously associated with the prevention of rapid HIV progression ${ }^{22}$, corroborating our findings that suggest that the intronic variant $r s 987106$ - $\mathrm{T}$ could be involved in CD $4^{+} \mathrm{T}$-cell restoration and thus to facilitate disease evolution. Regarding the $r s 3194051$ gene variant, a dual role has been identified. First, the AA genotype was related to higher pre-ART IL-7R values in IRs, and the H4 haplotype tagged by $r s 3194051$-A was associated with immune recovery status (15.5\% in IRs versus $10.3 \%$ in INRs), also suggesting protecting effect of this polymorphism. In agreement with this conclusion, allele $\mathrm{G}$ was previously found to be associated with susceptibility to MS, a chronic autoimmune T-cell mediated disease of the central nervous system in which T-cells play an important role ${ }^{28-30}$. Therefore, $r s 3194051-G$ SNP could potentiate an inflammatory status which is one of the main characteristic of MS risk, whereas $r s 3194051-A$ could confer some anti-inflammatory properties to IRs compared to INRs which are at higher risk of developing opportunistic infections. On the other hand, our results also indicated a recessive genetic effect (GG versus GA $+\mathrm{AA}$ ) relating the $\mathrm{G}$ allele to the absolute 
A
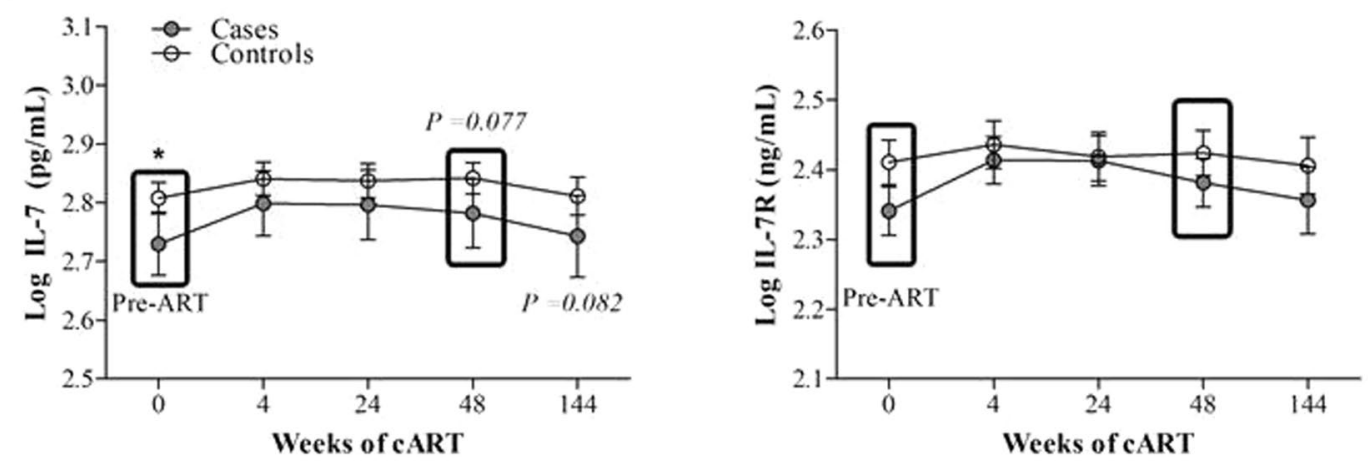

B
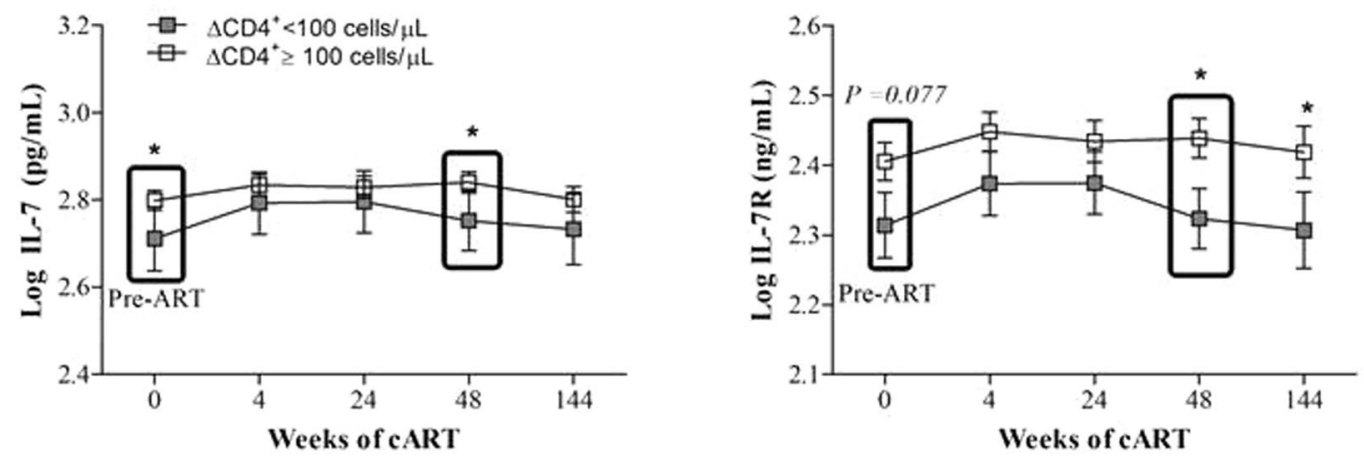

Figure 5. Longitudinal evaluation of the $I L-7 / I L-7 R$ axis during 144 weeks of ART. (A) Longitudinal study of circulating IL-7 and IL-7R values in the cases and the controls during 144 weeks of ART. (B) Longitudinal study of circulating IL-7 and IL-7R values in subjects classified according to the $\Delta \mathrm{CD} 4+\mathrm{T}$-cell count criterion during 144 weeks of ART. Data are represented as the mean \pm SEM Comparisons between groups were performed with nonparametric Mann-Whitney (MW) tests for unpaired samples and a Wilcoxon t-test for paired samples (W) (SPSS software).

increment of more than $100 \mathrm{CD} 4^{+} \mathrm{T}$-cells/ $\mu \mathrm{L}$. In accordance with this IHT model, the H2 haplotype tagged by rs3194051-G, which is more frequent than the aforementioned H4 haplotype tagged by $r s 3194051$-A, was associated with both successful immune recovery status ( $31.7 \%$ in IRs versus $27.4 \%$ INRs) and a $\triangle \mathrm{CD} 4^{+}$T-cell count of more than 100 cells $/ \mu \mathrm{L}$ ( $31 \%$ in $\Delta \mathrm{CD} 4^{+} \geq 100$ cells $/ \mu \mathrm{L}$ versus $26 \% \Delta \mathrm{CD} 4^{+}<100$ cells $/ \mu \mathrm{L}$ ). Consistent with these data, homozygous carriers of the G-allele at $r s 3194051$ experienced faster time to CD4 ${ }^{+}$T-cell count $>500$ cells/ $\mu \mathrm{L}$ compared with that of homozygous carriers of the A-allele subjects who were recruited from the Uganda AIDS Rural Treatment Outcomes (UARTO) cohort $^{19}$. Additionally, HIV/HCV coinfected patients with the $r s 3194051$ AA genotype showed a higher probability of severe liver fibrosis than did patients with the $r$ s 3194051 AG/GG genotype. The haplotype tagged by $r s 3194051$-A had higher odds of having advanced liver fibrosis than did the G haplotype HIV/HCV coinfected patients ${ }^{31}$, suggesting an association between the A allele and worse disease prognosis. Furthermore, the dissimilar genotype distributions of $r s 3194051$ are evidence of the modulation of the IL-7/IL-7R axis during ART and its role in the expression of the subpopulation of T-cells.

Moreover, regarding IL-7R gene variants, we detected CD4 ${ }^{+}$T-cell restoration after ART therapy in HIV subjects carrying the haplotypes tagged by $r s 1494558-\mathrm{G}, r s 969129-\mathrm{G}$ and $r s 1494555-\mathrm{T}$ (major allele of each SNP). Notice that these three IL-7R polymorphisms were previously related to each other ${ }^{31,32}$, which is not surprising due to the strong LD. Consistent with our data, $r$ s1494558-A was related to new-onset diabetes development after transplantation, playing an important role in the homeostasis of Treg cells, the regulatory $\mathrm{T}$ cells that play a major role in controlling immune responses to self-antigens ${ }^{33}$. Notice that diabetes is a metabolic complication associated with increased cardiovascular disease risk, a non-AIDS-defining clinical event, and INRs are associated with worse long-term clinical prognosis, including a higher risk of progression toward non-AIDS-defining clinical events $^{34}$. Later, the $r s 1494558$-A and $r s 1494555$-C genotypes were also associated with an increased risk of acute and chronic graft versus host disease after allogeneic hematopoietic cell transplantation ${ }^{35}$. In our study, haplotypes tagged by $r s 969129-\mathrm{G}$ and $r s 1494555$-T were related to immune restoration due to ART. In the case of $r s 14494555$, consistent with our data, a haplotype tagged by $r$ 1494555-C was referred to as the 'risk' haplotype because it was more prominent in MS patients compared with healthy donors, whereas the opposite effect has been observed for other haplotypes tagged by $r s 1494555-\mathrm{T}$, which has therefore been termed the 'protective' haplotype ${ }^{36}$.

Our study has some limitations. First, the number of patients per groups in genetic studies is not consistent with the number of available samples for the study of circulating plasma IL-7/IL-7R concentrations, which would make our results more consistent in the search of predictive and diseases progression markers. Additionally, 
specific data regarding the ART therapy would have been helpful to evaluate the implication of the different anti-retroviral drugs on circulating IL-7/IL-7R axis. Nevertheless, previous evidences suggest no differences between different drug families and IL-7/IL-7R axis.

In conclusion, taken together, our data suggest that IL-7/IL-7R mRNA expression and genotype could alter the homeostatic balance between soluble and membrane-bound receptors. We corroborate that IL-7R polymorphisms could affect T-cell homeostasis and function and that some IL-7R polymorphisms due to their position could influence other IL-7R polymorphisms, and combinations could impact immune pathologies. Further investigation of the IL-7/IL-7R axis could also elucidate new insights regarding T-cell reservoirs in HIV subjects.

\section{Patients and Methods}

Study design and participants. This was a multicentered, longitudinal case-controlled study comprising 416 adult HIV-infected subjects who were consecutively recruited between 2011 and 2013 at the HIV outpatient clinic of the participating hospitals and who started their first ART and achieved virological suppression after ART. Patients were selected from among those who were receiving a combination of two nucleoside reverse transcriptase inhibitors (NRTI) plus a non-nucleoside reverse transcriptase inhibitor (NNRTIs) or a protease inhibitor(s) (PI). A flow chart with patient selection and enrolment is provided in Fig. 1 and inclusion/exclusion criteria defined in Supplementary material. Of the selected patients, 215 were controls (baseline CD4 ${ }^{+} \mathrm{T}$-cell counts $>200$ cells $/ \mu \mathrm{L}$ ), and 201 were cases (baseline CD4 ${ }^{+} \mathrm{T}$-cell counts $\leq 200$ cells $/ \mu \mathrm{L}$ ). Among the cases, 106 subjects achieved more than $250 \mathrm{CD} 4^{+} \mathrm{T}$-cells/ $\mu \mathrm{L}$ after 48 weeks of ART ("immunological recoverers", IRs), and 95 subjects did not reach the 250 cells/ $\mu \mathrm{L} \mathrm{CD} 4^{+}$T-cell threshold ("immunological nonrecoverers", INRs). The absolute increment of $\mathrm{CD} 4^{+} \mathrm{T}$-cell counts was also calculated to re-classify HIV subjects into those that achieved more than $100 \mathrm{CD} 4^{+} \mathrm{T}$-cell counts/ $\mu \mathrm{L}$ after 48 of ART and those that did not. The study and all research protocols were carried out in accordance with the recommendations of the Ethical and Scientific Committees from each participating institution (Hospital Universitari de Tarragona Joan XXIII (Tarragona), Hospital de la Santa Creu i Sant Pau (Barcelona), Virgen del Rocío University Hospital (Seville), Hospital Clinic (Barcelona)) and were approved by the Committee for Ethical Clinical Research following the rules of Good Clinical Practice from the Institut d'Investigació Sanitària Pere Virgili (CEIm IISPV). The CEIm IISPV is an independent committee, made up of health and non-health professionals, which supervises the correct compliance of the ethical principles governing clinical trials and research projects that are carried out in our environment, specifically in its methodology, ethics and laws. All subjects gave written informed consent in accordance with the Declaration of Helsinki.

General laboratory measurements. Blood was drawn from a peripheral vein after an overnight fast. Whole blood was used to determine the $\mathrm{CD} 4^{+} \mathrm{T}$-cell count and for DNA isolation. Plasma was obtained by centrifugation and was stored at $-80^{\circ} \mathrm{C}$ until use. HIV-1 infection was diagnosed by a positive ELISA and confirmed by Western blot analysis. Plasma HIV-1 viral load was determined by the Cobas Amplicor HIV-1 Monitor Test v 1.5 (Roche Diagnostics, Barcelona, Spain). The limit of detectability is $<20$ copies/ $\mu \mathrm{L}$. CD $4^{+}$T-cell counts were analyzed using a flow cytometer FAC Scan (Becton Dickinson, San Jose, CA, USA).

Genetic studies. We selected 14 single nucleotide polymorphisms (SNPs) for IL7 and IL7R genes, with an allelic frequency greater than $20 \%$ in the Iberian Population in Spain (IBS) listed on the NCBI SNP database. In summary, the 14 SNPs analyzed in this study were the following for $I L-7: r s 6987789$ and $r s 7007634$; and for $I L-7 R$ : rs7701176, rs1494559, rs1494558, rs969128, rs969129, rs6893892, rs1494555, rs2228141 (rs7737000), rs6897932, rs987106, rs3194051 and $r s 10491434$.

Genomic DNA was extracted from peripheral blood with Qiagen kit (Qiagen, Hilden, Germany) and then the extracted DNA samples ( $5 \mathrm{ng} / \mu \mathrm{L}$ ) were sent to LGC Genomics Ltd. (formerly Kbioscience Ltd., Herts, UK) for genotyping. The number of individuals $(n=416)$ to be included in the genetic studies was at least 90 per group (Figs 1 and 4).

IL-7 and IL-7R plasma concentrations. Plasma concentrations of human IL-7 (log pg/mL) and IL-7R $(\log \mathrm{ng} / \mathrm{mL})$ were measured by double-antibody sandwich one-step process enzyme-linked immunosorbent assay (ELISA) QY-E04239 and QY-E04260, respectively (QAYEE-BIO, Shanghai, China), according to the manufacturer's instructions (Supplementary material).

Statistical analyses. Prior to the statistical analyses, the normal distribution and homogeneity of the variances were tested using a Kolmogorov-Smirnov test. Normally distributed data were expressed as the mean \pm standard deviation (SD), whereas variables with a skewed distribution were represented as the median $\left(25^{\text {th }}\right.$ percentile $-75^{\text {th }}$ percentile) or transformed into a decimal logarithm. Categorical variables were reported by number (percentages). Qualitative variables were analyzed using the $\chi 2$ test or Fisher's exact test when as necessary. Comparisons between groups were performed with nonparametric Kruskal-Wallis (KW) and/or Mann-Whitney (MW) tests for unpaired samples and a Wilcoxon t-test for paired samples (W). Associations between quantitative variables were evaluated using the Spearman correlation. Allele and genotype frequencies and Hardy-Weinberg equilibrium (HWE) were evaluated using SNPstats software ${ }^{37}$. To estimate the association between $I L-7$ and $I L-7 R$ genetic polymorphisms and immune recovery status, we use multiple inheritance models (codominant, dominant, recessive, overdominant and additive). For each SNP, odds ratios (ORs) and 95\% confidence intervals (CIs) were calculated using unconditional logistic regression analysis with adjustment of age and baseline pre-cART CD4+ T-cell counts. Statistical analyses were performed using SPSS (version 21.0, SPSS Inc., Chicago, IL), and graphical representations were generated with GraphPad Prism software (version 5.0, GraphPad Inc., San Diego, CA). The results were considered significant at $\mathrm{P}<0.05$. 


\section{Data availability}

The datasets used and/or analyzed during the current study are available from the corresponding author on reasonable request.

Received: 4 March 2019; Accepted: 11 October 2019;

Published online: 31 October 2019

\section{References}

1. Gaardbo, J. C., Hartling, H. J., Gerstoft, J. \& Nielsen, S. D. Incomplete immune recovery in HIV infection: Mechanisms, relevance for clinical care, and possible solutions. Clin. Dev. Immunol. 2012, 670957 (2012).

2. Battegay, M., Nüesch, R., Hirschel, B. \& Kaufmann, G. R. Immunological recovery and antiretroviral therapy in HIV-1 infection. Lancet Infect. Dis. 6, 280-287 (2006).

3. Goicoechea, M. et al. Determinants of CD4+ T cell recovery during suppressive antiretroviral therapy: Association of immune activation, T cell maturation markers, and cellular HIV-1 DNA. J. Infect. Dis. 194, 29-37 (2006).

4. Pacheco, Y. M. et al. Risk factors, CD4 long-term evolution and mortality of HIV-infected patients who persistently maintain low CD4 counts, despite virological response to HAART. Curr. HIV Res. 7, 612-619 (2009).

5. Loutfy, M. R. et al. A CD4+ cell count $<200$ cells per cubic millimeter at 2 years after initiation of combination antiretroviral therapy is associated with increased mortality in hiv-infected individuals with viral suppression. J. Acquir. Immune Defic. Syndr. 55, 451-459 (2010).

6. Pacheco, Y. M. et al. Increased risk of non-AIDS-related events in HIV subjects with persistent low CD4 counts despite cART in the CoRIS cohort. Antiviral Res. 117, 69-74 (2015).

7. Sieg, S. F. Interleukin-7 Biology in HIV Disease and the Path to Immune Reconstitution. Curr. HIV Res. 10, 341-347 (2012).

8. Ruiz-Mateos, E. et al. Endogenous IL-7 is associated with increased thymic volume in adult HIV-infected patients under highly active antiretroviral therapy. AIDS 17, 947-954 (2003).

9. Schluns, K. S., Kieper, W. C., Jameson, S. C. \& Lefrançois, L. Interleukin-7 mediates the homeostasis of naïve and memory CD8 T cells in vivo. Nat. Immunol. 1, 426-432 (2000).

10. Schluns, K. S. \& Lefrançois, L. Cytokine control of memory T-cell development and survival. Nat. Rev. Immunol. 3, 269-279 (2003).

11. Sheikh, V. et al. Administration of interleukin-7 increases CD4 T cells in idiopathic. Blood 127, 977-988 (2016).

12. Lévy, Y. et al. Effects of Recombinant Human Interleukin 7 on T-Cell Recovery and Thymic Output in HIV-Infected Patients Receiving Antiretroviral Therapy: Results of a Phase I/IIa Randomized, Placebo-Controlled, Multicenter Study. Clin. Infect. Dis. 55, 291-300 (2012).

13. Thiébaut, R. et al. Repeated Cycles of Recombinant Human Interleukin 7 in HIV-Infected Patients With Low CD4 T-Cell Reconstitution on Antiretroviral Therapy: Results of 2 Phase II Multicenter Studies. Clin. Infect. Dis. 62, 1178-1185 (2016).

14. Saidakova, E. V., Korolevskaya, L. B., Shmagel, N. G., Shmagel, K. V. \& Chereshnev, V. A. The role of interleukin 7 and its cell receptor in a poor recovery of CD4+ T cells in HIV-infected patients receiving antiretroviral therapy. Dokl. Biol. Sci. 458, 313-315 (2014).

15. Rallón, N. I. et al. Longitudinal assessment of interleukin 7 plasma levels in HIV-infected patients in the absence of and under antiretroviral therapy. J. Acquir. Immune Defic. Syndr. 58, 436-441 (2011).

16. Napolitano, L. A. et al. Increased production of IL-7 accompanies HIV-1 - mediated T-cell depletion: implications for T-cell homeostasis. Nat. Med. 7, 73-79 (2001).

17. Haas, D. W. et al. Immunogenetics of CD4 Lymphocyte Count Recovery during Antiretroviral Therapy: An AIDS Clinical Trials Group Study. J. Infect. Dis. 194, 1098-1107 (2006).

18. Rajasuriar, R. et al. Biological Determinants of Immune Reconstitution in HIV-Infected Patients Receiving Antiretroviral Therapy: The Role of Interleukin 7 and Interleukin 7 Receptor $\alpha$ and Microbial Translocation. J. Infect. Dis. 202, 1254-1264 (2010).

19. Rajasuriar, R. et al. The role of SNPs in the $\alpha$-chain of the IL-7R gene in CD4+ T-cell recovery in HIV-infected African patients receiving suppressive cART. Genes Immun. 13, 83-93 (2012).

20. Hartling, H. J. et al. Polymorphism in interleukin-7 receptor $\alpha$ gene is associated with faster $\mathrm{CD}^{+}{ }^{+} \mathrm{T}$-cell recovery after initiation of combination antiretroviral therapy. AIDS 28, 1739-1748 (2014).

21. Resino, S., Pérez, A., León, J. A., Gurbindo, M. D. \& Muñoz-Fernández, M. A. Interleukin-7 levels before highly active antiretroviral therapy may predict CD4+ T-cell recovery and virological failure in HIV-infected children. J. Antimicrob. Chemother. 57, 798-800 (2006).

22. Limou, S. et al. Identification of IL7RA Risk Alleles for Rapid Progression During HIV-1 Infection: A Comprehensive Study in the GRIV Cohort. Curr. HIV Res. 10, 143-150 (2012).

23. Ceredig, R. \& Rolink, A. G. The key role of IL-7 in lymphopoiesis. Semin. Immunol. 24, 159-164 (2012).

24. Song, H. et al. A three-base-deletion polymorphism in the upstream non-coding region of human interleukin 7 (IL-7) gene could enhance levels of IL-7 expression. Int. J. Immunogenet. 34, 107-113 (2007).

25. Lundmark, F., Duvefelt, K. \& Hillert, J. Genetic association analysis of the interleukin 7 gene (IL7) in multiple sclerosis. J. Neuroimmunol. 192, 171-173 (2007).

26. Guzmán-Fulgencio, M. et al. IL7RA polymorphisms predict the CD4+ recovery in HIV patients on cART. Eur. J. Clin. Invest 45, $1192-1199$ (2015).

27. Hutchinson, J. N. et al. Allele-specific methylation occurs at genetic variants associated with complex disease. PLoS ONE 9, e98464 (2014).

28. Liu, H. et al. Variants in the IL7RA gene confer susceptibility to multiple sclerosis in Caucasians: Evidence based on 9734 cases and 10436 controls. Sci. Rep. 7, 1207 (2017).

29. Zhang, Z. et al. Two genes encoding immune-regulatory molecules (LAG3 and IL7R) confer susceptibility to multiple sclerosis. Genes Immun. 6, 145-152 (2005).

30. Lundmark, F. et al. Variation in interleukin 7 receptor $\alpha$ chain (IL7R) influences risk of multiple sclerosis. Nat. Genet. 39, 1108-1113 (2007).

31. Lundtoft, C. et al. Autoimmunity risk- and protection-associated IL7RA genetic variants differentially affect soluble and membrane IL-7R $\alpha$ expression. J. Autoimmun. 97, 40-47 (2019).

32. Wang, J.-Y., Lin, C.-C., Lin, C. G.-J., Hsiao, Y.-H. \& Wu, L. S.-H. Polymorphisms of Interleukin 7 Receptor are Associated With MiteSensitive Allergic Asthma in Children in Taiwan. Tzu Chi Med. J. 22, 18-23 (2010).

33. Kim, Y. G. et al. Association of genetic polymorphisms of interleukins with new-onset diabetes after transplantation in renal transplantation. Transplantation 93, 900-907 (2012).

34. Rosado-Sánchez, I. et al. Glutaminolysis and lipoproteins are key factors in late immune recovery in successfully treated HIVinfected patients. Clin. Sci. 133, 997-1010 (2019).

35. Shamim, Z. et al. Polymorphism in the Interleukin-7 Receptor-alpha and Outcome after Allogeneic Hematopoietic Cell Transplantation with Matched Unrelated Donor. Scand. J. Immunol. 78, 214-220 (2013).

36. Jäger, J., Schulze, C., Rösner, S. \& Martin, R. IL7RA haplotype-associated alterations in cellular immune function and gene expression patterns in multiple sclerosis. Genes Immun. 14, 453-461 (2013).

37. Solé, X., Guinó, E., Valls, J., Iniesta, R. \& Moreno, V. SNPStats: A web tool for the analysis of association studies. Bioinformatics 22, 1928-1929 (2006). 


\section{Acknowledgements}

This study would not have been possible without the collaboration of all the patients and medical and nursing staff who have taken part in the project. This work was supported by the Fondo de Investigacion Sanitaria [PI10/02635, PI13/0796, PI14/1693, PI16/00503, PI16/00684, PI19/01337]-ISCIII-FEDER; Programa de Suport als Grups de Recerca AGAUR (2014SGR250, 2017SGR948); Gilead Fellowship Program GLD14/293 and GLD17/00299; The SPANISH AIDS Research Network [RD12/0017/0005, RD16/0025/0002, RD16/0025/0006, RD16/0025/0019, RD16/0025/0020]-ISCIII-FEDER (Spain) and Consejería de Economía, Innovación, Ciencia y Empleo, Junta de Andalucía (Proyecto de Investigación de Excelencia; CTS2593). FV is supported by grants from the Programa de Intensificación de Investigadores (INT15/226)-ISCIII. YMP and ERM are supported by the Servicio Andaluz de Salud through Programa Nicolás Monardes (C-0013/17 and C-0032/17, respectively). E.R-M was also supported by Programa Miguel Servet ISCIII, FEDER, CPII014/00025. AR is supported by a grant from the Acció Instrumental d'incorporació de científics i tecnòlegs (PERIS SLT002/16/00101), Departament de Salut, Generalitat de Catalunya and Agencia Estatal de Investigación (Acciones de carácter internacional "Europa Investigación”; EUIN2017-89297), Ministry of Economy and Competitiveness. We are also grateful to GESIDA for "Premio para Jóvenes Investigadores 2019" to AR. Authors greatly appreciate the comments and criticisms of the anonymous reviewers that greatly helped to improve the manuscript.

\section{Author contributions}

All authors have seen and approved the submitted version of the manuscript. The authors contributions are as follows: experimental design (A.C., E.R., M.L.D., A.R.) and intellectual guidance (J.P., C.V., Y.M.P., E.R.M.); recruitment of subjects (J.P., M.L.D., P.D., C.V., J.V.G., M.P., C.P., S.V., J.M.) and sample procurement (V.A., M.V., A.J.C.); data collection (A.C., E.R.); data analysis and interpretation (E.R.G., E.R.M., Y.M.P., A.R.); manuscript preparation (A.C., E.R., F.V., A.R.). A.C., E.R., F.V. and A.R. were responsible for the study design, data analysis, and article development. F.V. and A.R. reviewed and edited the manuscript.

\section{Competing interests}

The authors declare no competing interests.

\section{Additional information}

Supplementary information is available for this paper at https://doi.org/10.1038/s41598-019-52025-8.

Correspondence and requests for materials should be addressed to F.V.

Reprints and permissions information is available at www.nature.com/reprints.

Publisher's note Springer Nature remains neutral with regard to jurisdictional claims in published maps and institutional affiliations.

Open Access This article is licensed under a Creative Commons Attribution 4.0 International License, which permits use, sharing, adaptation, distribution and reproduction in any medium or format, as long as you give appropriate credit to the original author(s) and the source, provide a link to the Creative Commons license, and indicate if changes were made. The images or other third party material in this article are included in the article's Creative Commons license, unless indicated otherwise in a credit line to the material. If material is not included in the article's Creative Commons license and your intended use is not permitted by statutory regulation or exceeds the permitted use, you will need to obtain permission directly from the copyright holder. To view a copy of this license, visit http://creativecommons.org/licenses/by/4.0/.

(C) The Author(s) 2019 